\title{
Advances in MBE Selective Area Growth of III-Nitride Nanostructures: From NanoLEDs to Pseudo Substrates
}

\author{
Steven Albert , Ana Maria Bengoechea-Encabo , Francesca Barbagini, David Lopez-Rormero, \\ Miguel Angel Sanchez-Garcia and Enrique Calleja
}

\author{
Pierre Lefebvre
}

Xiang Kong, Uwe Jahn and Achim Trampert

Marcus Müller, Frank Bertram, Gordon Schmidt, Peter Veit, Silke Petzold and Jürgen Christen

Philippe De Mierry and Jesus Zuñiga-Perez

The aim of this work is to provide an overview on the recent advances in the selective area growth (SAG) of (In)GaN nanostructures by plasma assisted molecular beam epitaxy, focusing on their potential as building blocks for next generation LEDs.

The first three sections deal with the basic growth mechanisms of GaN SAG and the emission control in the entire ultraviolet to infrared range, including approaches for white light emission, using InGaN disks and thick segments on axial nanocolumns. SAG of axial nanostructures is developed on both GaN/sapphire templates and GaN-buffered Si(111).

As an alternative to axial nanocolumns, section 4 reports on the growth and characterization of InGaN/GaN core-shell structures on an ordered array of top-down patterned GaN microrods. Finally, section 5 reports on the SAG of GaN, with and without InGaN insertion, on semi-polar (11-22) and non-polar (11-20) templates. Upon SAG the high defect density present in the templates is strongly reduced as indicated by a dramatic improvement of the optical properties. In the case of SAG on nonpolar (11-22) templates, the formation of nanostructures with a low aspect ratio took place allowing for the fabrication of high-quality, non-polar $\mathrm{GaN}$ pseudo-templates by coalescence of these nanostructures.

Keywords: Selective area growth; nanostructures; InGaN; GaN; LED; photoluminescence; white-light emission; single color emission; core-shell; non-polar; semi-polar; pseudo substrates; coalescence. 


\section{Introduction}

Light emitting diodes (LEDs) are anticipated to have great potential as a replacement for traditional lamp-based lighting systems due to a higher efficiency, with the greatest expected impact for high-quality white-light sources. The main white light source up to now is the incandescent light bulb which converts only about $5 \%$ of electricity into visible light. Due to that lighting is the second largest user of energy in in-house lighting ${ }^{1}$ causing greenhouse gas emissions of 1900 Megatons of $\mathrm{CO}_{2}$ per year ${ }^{2}$. That corresponds to over three times the emissions caused by aircraft traffic ${ }^{3}$. All these drawbacks led to a gradual ban of incandescent light bulbs by many governments.

The strongest two competitors as a replacement for incandescent lamps are compact fluorescent lamps (CFLs) and non-organic LEDs. Due to the relatively high price of nonorganic LEDs, CFLs are the dominant replacement for general household lighting at the moment. They have a four times higher efficiency than incandescent bulbs and a lifetime of up to $10000 \mathrm{~h}$ (compared to $1000 \mathrm{~h}$ for incandescent light bulbs). A drawback of CFLs is the use of mercury which is highly toxic. This can apparently be considered as a severe issue considering the fact that most states already banned mercury thermometers. In addition the efficacy of CFLs is not expected to exceed $100 \mathrm{~lm} / \mathrm{W}$. Due to that there is a high need to develop highly efficient white light sources that do not contain any toxic materials, making non-organic GaN-based LEDs the perfect lamp choice for the future. Unlike every conventional light source, LEDs directly transfer electrical energy into light. The most common approach for white light generation using LEDs is a blue LED that pumps phosphors that emit at longer wavelengths, i.e. phosphor converted LEDs (pc-LEDs). The simplest pc-LEDs combine a blue LED $(\lambda=440-460 \mathrm{~nm})$ with a YAG: $\mathrm{Ce}^{3+}$ phosphor $(\lambda=560)$. The problem with that approach is a rather low color rendering index (CRI) of 70-80 and a correlated color temperature (CCT) of 4000 to $8000 \mathrm{~K}$. With these characteristics these LEDs are only suitable for less demanding applications such as outdoor lighting. For indoor applications CRIs between 80 and 90 are needed ${ }^{4}$. The figures of merit of a pc-LED can be improved by adding a second redemitting phosphor such as nitrodosilicate. With this approach commercial white LEDs with CRIs of 90 , efficacies of $55 \mathrm{~lm} / \mathrm{W}$ and CCTs of 3000-6000K have already entered the market. An alternative to pc-LEDs is the RG(Y)B approach in which solely LEDs are used for white light generation. With this approach CRIs higher than 95 as well as increased efficiencies can be achieved ${ }^{5,6}$. With the RGB approach a dynamic color control is possible that would allow for a real-time spectral tuning. As a main advantage over pc-LEDs, an efficiency improvement due to the absence of color filters or phosphors is expected. Assuming an efficiency of around $25 \%$ for each part, efficacies of around $160 \mathrm{~lm} / \mathrm{W}$ are theoretically possible. The disadvantages of this approach up to now are different efficiencies as well as temperature dependencies of the efficiencies when using different material systems such as GaAlInP materials for the red and InGaN materials for the blue and green, leading to unacceptable variations of the color unless compensated by current drivers and feedback loops for each color which would add extra cost. 
The two main obstacles to overcome, before large scale commercialization of RGBLEDs, are; i) the green gap problem and ii) the efficiency droop of InGaN LEDs which refers to a decrease of efficiency with increasing injection current. The green gap problem refers to the fact that efficient LED emission could not be demonstrated yet across the entire visible spectrum, particularly from green to yellow. A good performance can only be achieved in the blue range using InGaN based LEDs and red range using AlGaInP based LEDs. The efficiency drop of AlGaInP based materials at shorter wavelengths is caused by an indirect band gap crossover in the green-yellow range which can hardly be solved. A solution may be the use of InGaN for bridging the green-yellow gap since it has a direct bandgap across the complete visible range with no intrinsic roadblock to high efficiency optical emission. Up to now most research effort has been focused on InGaN/GaN based c-plane oriented, two-dimensional quantum well (QW) structures. In these planar structures the green gap problem is assumed to be caused by: (i) the reduction of the radiative recombination rate induced by the quantum confined Stark effect due to spontaneous and piezoelectric polarizations, and (ii) the high density of non-radiative defects due to the increasing lattice mismatch (strain) between GaN and InGaN alloys with increasing In content. The strong polarization effects have their origin in the wurtzite crystal structure leading to piezoelectric and spontaneous polarization along the polar [0001] crystal direction causing large ( $>1 \mathrm{MV} / \mathrm{cm}$ ) electrostatic fields in the QW. At this point it has to be emphasized that the piezoelectric polarization depends on strain, leading to a reduction in radiative recombination rate for higher In contents assuming that InGaN is grown coherently strained on GaN.

In addition to its impact on the polarization, strain also causes a number of additional effects. In general the growth of InGaN requires a rather low growth temperature due to the reduced thermal stability of $\mathrm{InN}^{7}$. At a given growth temperature the strain in an InGaN/GaN heterostructure reduces the incorporation of indium due to compositional lattice pulling effects ${ }^{8,9}$. Due to that even lower growth temperatures are needed in order to achieve higher In content. The combination of a low growth temperature and an increasing strain energy leads to the formation of a number of structural defects, e.g. point defects ${ }^{10}$, incorporation of impurities ${ }^{11}$ and V-defects ${ }^{12,13}$ causing a decrease of the optical quality of the active region.

A potential solution for all problems discussed above may be the use of ordered nanocolumnar structures as building blocks for next generation LEDs. It has been shown several years ago that dislocation- and strain- free group-III nitrides can be grown on $\mathrm{Si}(100)$ and (111), as well as on amorphous $\mathrm{SiO}_{2}$ substrates in the form of one-dimensional structures in a self-assembled fashion ${ }^{14-18}$. When growing nanocolumnar InGaN/GaN heterostructures a higher strain can be accommodated (due to the strain relief by lateral relaxation) before dislocations generate ${ }^{19}$. However, LEDs based on InGaN/GaN selfassembled nanocolumns (NCs) always show polychromatic emission that derives from an inhomogeneous axial and radial strain and In\% distribution, geometry dispersion, and an inherent tendency of InGaN alloys to develop composition fluctuations ${ }^{20,21}$. In addition, efficient and reliable LEDs based on self-assembled NCs are hindered by limitations due to strong electrical characteristics dispersion ${ }^{22}$. 
In order to overcome these limitations, selective area growth (SAG) of GaN ${ }^{23-26}$ has been developed. It allows the growth of NCs with well-controlled position and diameter, resulting in periodic arrays of NCs with very little morphology dispersion.

This review summarizes recent advances of Calleja et al. in the selective area growth of III-V nanostructures by plasma assisted molecular beam epitaxy (PAMBE) and is divided into six sections.

The first section of this work addresses the ordered growth of GaN NCs, with and without InGaN nano-disks, at different temperatures in order to study morphology changes and their influence on the optical properties of these nanostructures. During SAG of GaN NCs on c-plane GaN templates, the growth front (topside) is generally formed by semipolar planes and a topmost c-plane ("pencil-like" profile).

The second section reports on the SAG of InGaN/GaN nanocolumnar structures on GaN/sapphire templates in order to explore their potential for LED applications. InGaN NCs emitting in the red, green, and blue spectral range have been fabricated. White light emission at room temperature was achieved by either using composition-graded InGaN active regions or by a monolithic integration of red, green, and blue emitting InGaN sections in each NC. In addition as a first step towards the fabrication of actual NC based white light emitters, single color emission from nanocolumnar pin diodes is demonstrated.

A very important issue related to commercial LED applications is the fabrication of ordered InGaN nanocolumnar structures directly on cheap, high quality substrates, like $\mathrm{Si}$, a semiconductor having a well established processing technology. The growth of InGaN/GaN NCs with In contents up to $100 \%$ on GaN-buffered Si(111) substrates will be presented in section 3 .

As an alternative approach to axial structures, the growth of InGaN/GaN core-shell structures has been proposed and achieved some years ago by MOVPE, in order to take advantage of the large sidewall active area and the non-polar character of the $\mathrm{m}$-planes that greatly enhances emission or absorption in respect to the axial case ${ }^{27-33}$. Most InGaN/GaN core-shell columnar heterostructures have micron-size diameter and height, rather than nanoscale dimensions, in order to largely increase the active area. The fourth section of this review discusses the growth by PAMBE of InGaN/GaN core-shell structures on selectively etched GaN microrods using a combination of top-down principle (microrod patterned GaN/sapphire substrate) and the bottom-up (epitaxy) process in a conformal way around the patterned microrods.

A solution for the increased piezoelectric polarization with increasing In content, is the use of non-polar (m-plane (1-100) or a-plane (11-20)) or semipolar (11-22) crystal orientations. Up to now, the best results were obtained using non polar or semi polar bulk substrates. An alternative approach is the use of SAG. The fifth section presents results on SAG of GaN nanostructures, grown with and without an insertion of InGaN, on semi-polar (11-22) and non-polar (11-20) GaN templates. It will be shown that due to SAG on (11-22) GaN most of the basal stacking faults (BSFs) present in the initial GaN template are filtered, drastically improving the optical quality of the nanostructures. Furthermore, when embedding an InGaN insertion into the nanostructures grown on semi-polar GaN, the In incorporation is shown to strongly depend on the crystallographic orientation, being 
significantly larger at the apex of the nanostructures (polar and semi-polar facets) than on the lateral non-polar facets. In addition the fabrication of high quality non-polar pseudotemplates using coalesced nanostructures on non-polar (11-20) GaN templates as an alternative to high cost bulk GaN substrates is presented.

The PAMBE system used was equipped with a rf-plasma source providing active nitrogen and standard Knudsen cells for $\mathrm{Ga}, \mathrm{In}, \mathrm{Si}$ and $\mathrm{Mg}$. Samples were grown on polar (0001), non-polar (11-20) or semi-polar (11-22) GaN/sapphire or Si(111) substrates using a nanohole titanium mask fabricated by either e-beam lithography or colloidal lithography in case of bottom up SAG, and on selectively etched GaN pillars in case of core-shell growth. Metal and nitrogen fluxes were calibrated in (0001) GaN and (0001) InN growth rate units $(\mathrm{nm} / \mathrm{min})$. In wurtzite $\mathrm{GaN}$ and $\mathrm{InN}$ the areal densities referring to 1 monolayer (ML) are $1.14 \times 10^{15} \mathrm{GaN} / \mathrm{cm}^{2}$ and $9.17 \times 10^{14} \mathrm{InN} / \mathrm{cm}^{2}$ respectively. Once grown, the samples were studied with scanning electron microscopy (SEM), photoluminescence (PL), transmission electron microscopy (TEM), atomic force microscopy (AFM), cathodoluminescence (CL), and capacitance-voltage (C-V) using a Hg probe.

\section{Results and Discussion}

\subsection{Morphology control of selectively grown GaN Nanocolumns: Impact on the optical properties of InGaN insertions}

A series of GaN NCs samples was gown on GaN/sapphire templates for $1 \mathrm{~h}$ at $940^{\circ} \mathrm{C}, 900^{\circ} \mathrm{C}$ and $860^{\circ} \mathrm{C}$ in order to study the effects of growth temperature on the NC morphology and to better understand the driving mechanisms of SAG. The gallium $\left(\Phi_{\mathrm{Ga}}\right)$ and nitrogen $\left(\Phi_{\mathrm{N}}\right)$ fluxes used were $15 \mathrm{~nm} / \mathrm{min}$ and $5 \mathrm{~nm} / \mathrm{min}$, respectively for the three samples. An additional GaN NC sample was grown at $900^{\circ} \mathrm{C}$ with a $\Phi_{\mathrm{Ga}}=18 \mathrm{~nm} / \mathrm{min}$ keeping the same $\Phi_{\mathrm{N}}$ to determine the effects of the III/V ratio on morphology.

The dependence of the optical properties on the NCs morphology was studied in a second series of SAG NC GaN samples, having either pencil-like or flat top, where a single InGaN nano-disk was embedded. The nano-disks were grown in all cases at $625^{\circ} \mathrm{C}$ with $\Phi_{\mathrm{N}}=14 \mathrm{~nm} / \mathrm{min}, \Phi_{\mathrm{Ga}}=4.3 \mathrm{~nm} / \mathrm{min}$, and $\Phi_{\mathrm{In}}=4.3 \mathrm{~nm} / \mathrm{min}$. The nano-disk was capped with a GaN layer (20 to $40 \mathrm{~nm}$ thick) grown under the same conditions used for the InGaN nanodisk (closing the In flux). More details about the results presented in this section can be found in reference 34 .

When comparing the SAG of GaN NCs on GaN/sapphire templates with the classical self-assembled growth of GaN NCs on $\mathrm{Si}(111)$, the most obvious differences are a much higher temperature and III/V ratio used for the SAG. A much higher temperature is required to enhance Ga atoms diffusivity on the mask to reach the nanoholes while preventing GaN nucleation on the mask by Ga desorption. This forces an increase in the $\Phi_{\mathrm{Ga}}$ because the overall Ga desorption, including from the GaN nanoholes where the SAG is intended, increases exponentially with growth temperature. On the other hand, too much nitrogen increases the probability to nucleate GaN on the mask at a given temperature before the Ga ad-atoms desorb or diffuse into the nanoholes. Moreover, an increase of nitrogen enhances 
the energy barrier for Ga diffusion which in turn further increases the probability to nucleate GaN on the mask. Both mechanisms lead to a loss of selectivity. Thus, adequate (high) growth temperature and $\Phi_{\mathrm{Ga}} / \Phi_{\mathrm{N}}$ are needed to reach selectivity. Once the selectivity of growth was achieved, the substrate temperature and III/V ratio will also affect the development of NCs within each nanohole, as well as their shape, as shown in figure 1.

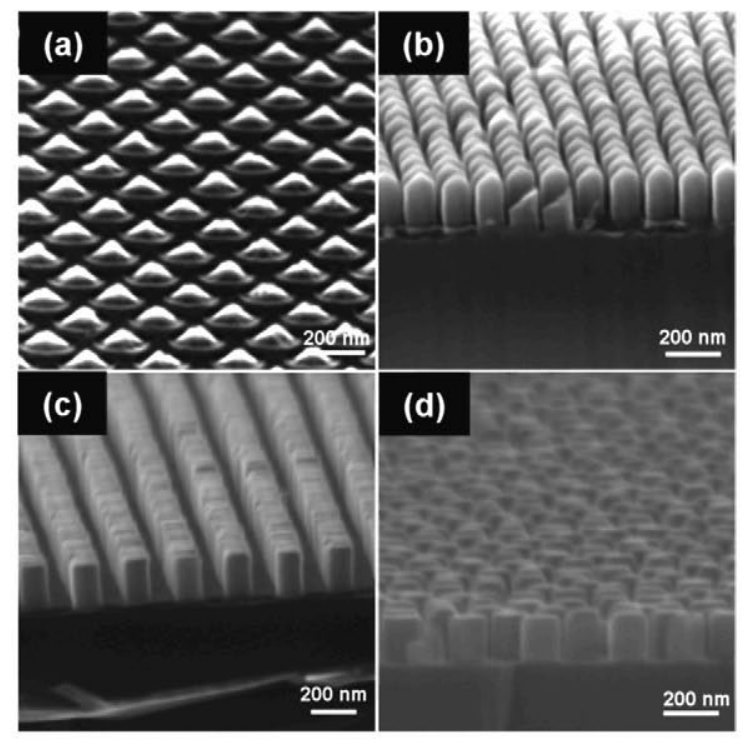

Fig. 1. SEM images of different morphologies of ordered GaN NCs grown under the same nominal III/V ratio of 3 but different temperatures; (a) $940^{\circ} \mathrm{C}$, (b) $900^{\circ} \mathrm{C}$, and (c) $860^{\circ} \mathrm{C}$. In (d), flat top ordered GaN NCs grown at $900^{\circ} \mathrm{C}$, like in (b), but under a nominal III/V ratio of 3.6, Appl. Phys. Lett. 100, 231906 (2012).

If the temperature is too high (figure 1.a) Ga desorption is high and the remaining Ga within the nanoholes is sufficient to start SAG but not to develop vertical sidewalls (no NC growth). Selectivity is achieved and it can be observed that $r$-facets develop in the nanocrystals within each nanohole (pyramid-like). There is a significant transient of the $\Phi_{\mathrm{Ga}}$ upon shutter opening which is assumed to yield a $\Phi_{\mathrm{Ga}}$ high enough, for a short period of time, to nucleate GaN on each nanohole. Once the transient is over, the reduced stable $\Phi_{\mathrm{Ga}}$ is not sufficient to sustain $\mathrm{NC}$ growth.

The shortage of $\Phi_{\mathrm{Ga}}$, as responsible for the above mentioned growth stop, is supported by results shown in figure $1 \mathrm{~b}$ corresponding to a sample grown at lower $\left(40^{\circ} \mathrm{C}\right)$ temperature. In this case, Ga desorption is exponentially reduced, or, in other words, the available Ga within each nanohole is enough to allow growth until GaN NCs develop keeping their pyramidal top shape (pencil-like). A further reduction of the growth temperature, down to $860^{\circ} \mathrm{C}$, keeps selectivity and allows NCs growth, though their top shape is now flat (c-plane, in figure 1c). This change in NC top morphology is attributed to an increase of the local III/V ratio within each nanohole that avoids the development of $\mathrm{r}$-facets from the growth beginning. A similar result is obtained keeping the temperature at $900^{\circ} \mathrm{C}$ but increasing the nominal III/V ratio (from 3 to 3.6 ) as shown in figure 1.d. This 
confirms the hypothesis that a high enough local III/V ratio within each nanohole (either by reducing desorption or by increasing the nominal $\Phi_{\mathrm{Ga}}$ ) leads to GaN NCs with a flat top (polar c-plane), while a smaller local III/V ratio will lead to a pyramidal top morphology. We can speculate that the transition from pyramidal to flat top reflects a change from Nrich to Ga-rich growth, taking into account that the local Ga flux ( $\left.\Phi_{\text {localGa }}\right)$ on the nanoholes would be $\Phi_{\mathrm{Ga}}-\Phi_{\mathrm{dGa}}(\mathrm{T})+\Phi_{\mathrm{Gadff}}\left(\mathrm{T}\right.$, mask design), where $\Phi_{\mathrm{dGa}}$ is the desorption flux (growth temperature dependent), and $\Phi_{\text {Gadff }}$ is the diffusion flux. These findings agree with results for the homoepitaxial growth of GaN layers, where the surface morphology is determined by the growth regime (Ga-rich or N-rich) with $\mathrm{N}$-rich conditions yielding a faceted surface ${ }^{35,36}$. It is worth noting that the GaN NCs SAG is a homoepitaxial growth of GaN on the GaN areas defined by the masking openings.

These findings are summarized in a plot shown in figure 2. Assuming selective conditions, it is shown that for a fixed $\Phi_{\mathrm{N}}$ the ratio $\Phi_{\text {localGa }} / \Phi_{\mathrm{N}}$ determines the morphology during the SAG, with $\Phi_{\text {localGa }}$ being dependent on the growth temperature (left axis) and Ga impinging flux. For simplicity reasons no geometrical effects (mask), that were found to play a role as well ${ }^{26}$, are considered here.

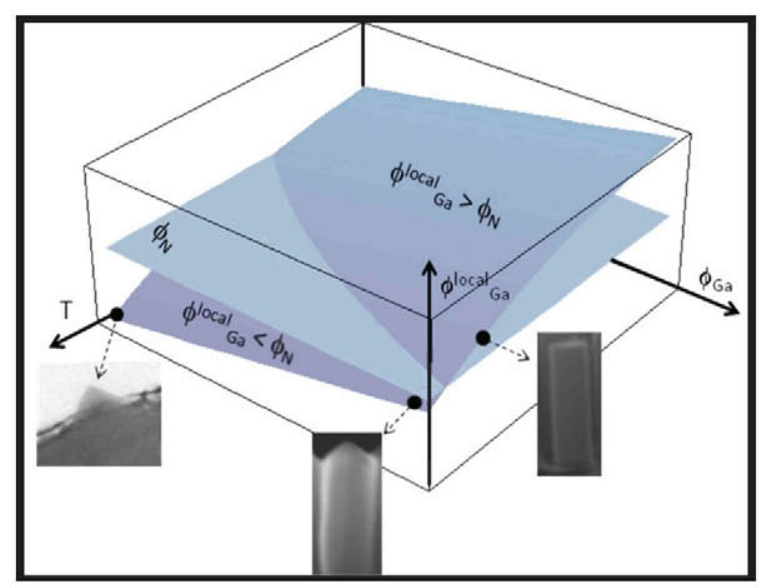

Fig. 2. Impact of growth temperature and Ga-flux on the GaN NC morphology during SAG; for a fixed $\Phi_{\mathrm{N}}$ value, the ratio $\Phi_{\text {localGa }} / \Phi_{\mathrm{N}}$ (we speculate: $>1$ flat top, $<1$ pencil-like top) is mainly controlled by growth temperature and impinging Ga flux $\Phi_{\mathrm{Ga}}$. adapted from $\mathrm{A}$. Bengoechea-Encabo, "Crecimiento, fabricación y caracterización de heteroestructuras y nanocolumnas ordenadas basadas en nitruros del grupo III para aplicaciones sensoras", $\mathrm{PhD}$ thesis, 2012.

As pointed out in the introduction, the performance of current QW based LEDs is limited in the green (green gap) range, most likely due to strong polarization fields. The ability to control the top morphology of the GaN NCs allows the growth of InGaN QWlike structures mostly along semi-polar directions, though the overall NC growth proceeds along the polar c-direction. This would lead to a significantly reduced internal field within the InGaN QW structure. The potential of this kind of structure is explored in samples where InGaN nanoDisks are included into the ordered GaN NCs with flat and pyramidal top morphologies. 


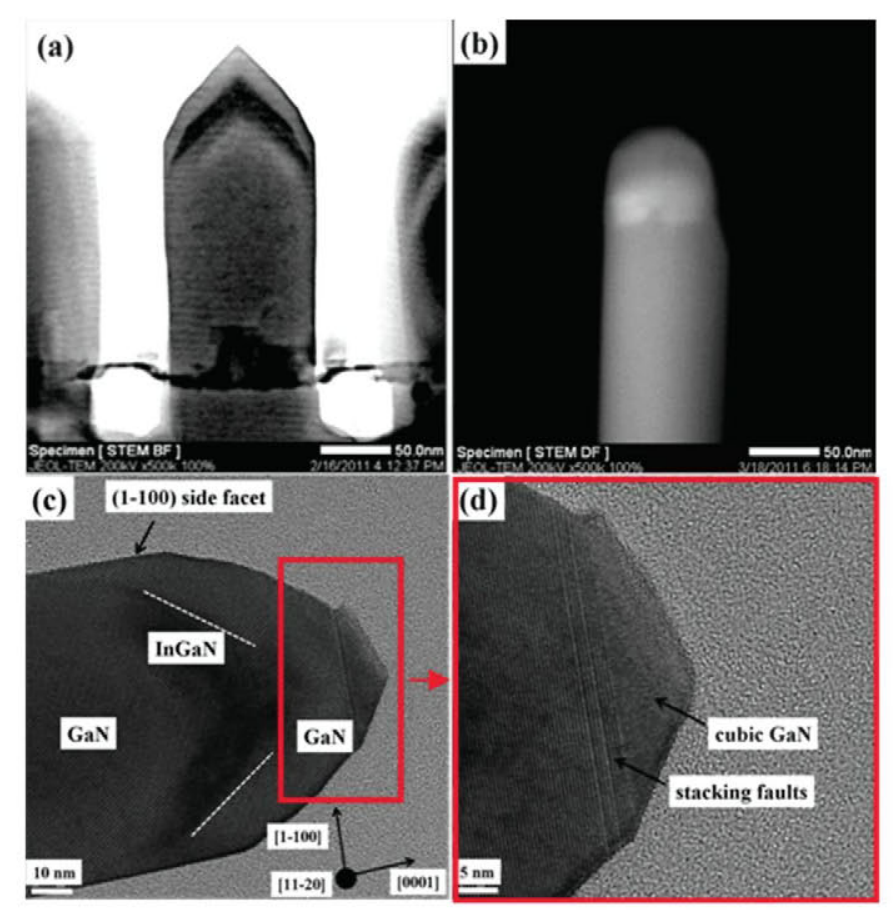

Fig. 3. (a) STEM bright-field image of InGaN nanoDisk embedded in GaN NCs with pencil like top; (b) HAADFSTEM image of an InGaN nanoDisk embedded in GaN NCs with flat top; (c) and (d) HRTEM images of the embedded InGaN nanoDisk shown in (a). adapted from Appl. Phys. Lett. 100, 231906 (2012).

As shown in figures $3 a$ and $3 b$, the morphology of the InGaN embedded nanoDisks is determined by the top morphology of the GaN NC underneath. Whereas a true disk geometry is obtained in flat top NCs, an inverted V-shaped InGaN with semipolar planes (r-plane and other ones) is found in pyramidal top NCs. The NCs structure is free of defects but for the topmost GaN cap layer, where stacking faults and cubic inclusions are found, most likely due to a low growth temperature (figures $3 \mathrm{c}$ and $3 \mathrm{~d}$ ).

Low temperature PL spectra shown in figure 4 correspond to samples where the InGaN "nanoDisks" were grown during the same time $(160 \mathrm{sec})$ corresponding to a nominal thickness of $25 \mathrm{~nm}$, though the different crystal planes involved in the pyramidal top case may produce changes in both thickness and In\%. Figure 4 shows a strong PL peak energy shift between the two samples $(0.4 \mathrm{eV})$ which can be explained by either: i) different In\% related to crystal planes (c-plane vs semi polar ones), or ii) different strain state and internal electric fields, again related to polar and semipolar planes.

The PL spectra also show strong intensity differences being much lower (1/43) in the case of the NCs with flat top that can be related to the stronger internal electric field in case of flat top NCs (c-plane) that would lead to a weaker electron-hole wavelength overlapping, while this field would be very much reduced in the case of semipolar planes ${ }^{37,38}$. As mentioned before, a smaller In\% incorporation in semipolar planes would also add to the blue shift observed in pyramidal NCs. 


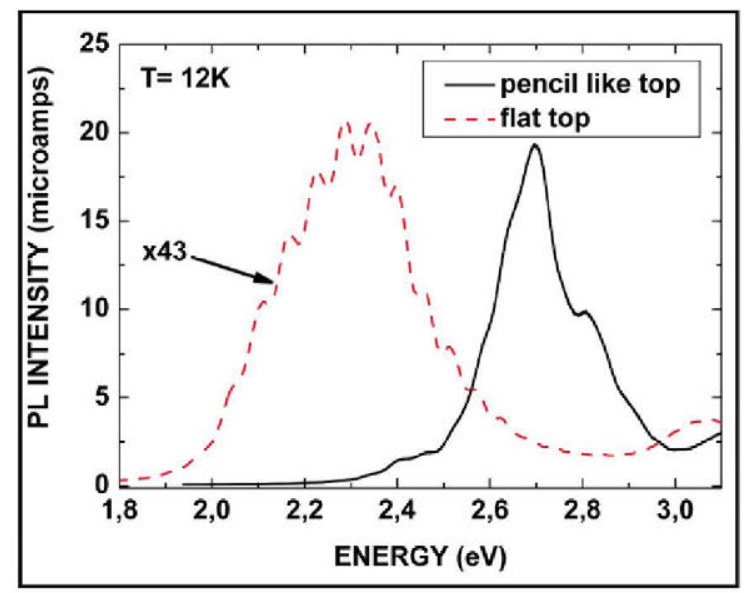

Fig. 4. LT- PL spectra of two GaN/InGaN/GaN ordered nanocolumnar heterostructures with InGaN regions having flat and pyramidal shape. Appl. Phys. Lett. 100, 231906 (2012).

To sum up it has been shown that depending on the growth conditions, three growth regimes can be distinguished being i) GaN pyramids with no further vertical growth; ii) GaN NCs with top semipolar planes; and iii) GaN NCs with a top c-plane. The driving mechanism for the change of morphology is the actual local III/V ratio within the nanohole mask.

The effects of these morphology changes on the optical properties of InGaN nanoDisks has been assessed by PL measurements, that show a strong red shift and reduced PL emission intensity for the InGaN nanoDisks grown on flat top (c-plane) GaN NCs. This shift is interpreted in terms of a lower In $\%$ and electric fields on semipolar planes.

\subsection{Selective area growth of InGaN nanocolumns on GaN/sapphire substrates}

The attempt to grow InGaN NCs directly on GaN/sapphire templates is considered extremely challenging due to the competing requirements of In incorporation and selectivity. A low substrate temperature is needed in order to minimize InN decomposition and In desorption, whereas a higher temperature is needed to achieve selectivity, as discussed in section 1. In order to circumvent this problem, $600 \mathrm{~nm}$ long ordered GaN NCs were first grown, allowing the subsequent growth of InGaN regions at adequate temperatures. This method prevents the nucleation of InGaN on the mask during the InGaN growth due to shadowing effects by the long GaN NCs. The two step process for GaN NCs was: i) 1 hour growth of $\mathrm{GaN}$ at $880^{\circ} \mathrm{C}$ with $\Phi_{\mathrm{Ga}}=16 \mathrm{~nm} / \mathrm{min}$ and $\Phi_{\mathrm{N}}=5 \mathrm{~nm} / \mathrm{min}$ in order to ensure perfect selectivity with a growth rate as small as $3 \mathrm{~nm} / \mathrm{min}$; ii) 1 hour growth of GaN at $840^{\circ} \mathrm{C}$ with $\Phi_{\mathrm{Ga}}=8 \mathrm{~nm} / \mathrm{min}$ and $\Phi_{\mathrm{N}}=14 \mathrm{~nm} / \mathrm{min}$ to increase the growth rate. More details about this growth method are found in references 39,40 .

This section will show results on the achievement of blue, green and red emission from nanocolumnar InGaN/GaN structures by changing the growth temperature, In/Ga ratio and III/V ratio. For this purpose, a series (A) of six samples (T1-T6) was grown under the 
conditions described in table 1 . In addition, results in this section will demonstrate white light emission from nanocolumnar InGaN/GaN structures using either graded or RedGreen-Blue (RGB) InGaN stacked structures. In the case of composition-grading, two samples $(\mathrm{T} 7, \mathrm{~T} 8)$ were grown, while in the case of RGB approach, another two samples $(\mathrm{T} 9, \mathrm{~T} 10)$ were grown using stacked regions of InGaN grown under the same conditions as T4, T5, and T6. In sample T9 each InGaN region was grown for $20 \mathrm{~min}$, whereas in sample T10 the growth times of each region were changed to "tailor" the emission spectrum.

Table 1. Growth conditions for the InGaN/GaN NCs.

\begin{tabular}{|c|c|c|c|c|c|c|}
\hline \multirow{2}{*}{$\begin{array}{l}\text { sample } \\
\text { number }\end{array}$} & \multicolumn{3}{|c|}{$\begin{array}{l}\text { impinging fluxes in } \mathrm{nm} / \mathrm{min} \\
\left(\times 10^{14} \text { atoms } / \mathrm{s} \mathrm{cm}^{2}\right)\end{array}$} & \multirow{2}{*}{ III/N ratio } & \multirow{2}{*}{$\begin{array}{l}\ln / \mathrm{Ga} \\
\text { ratio }\end{array}$} & \multirow{2}{*}{$\begin{array}{l}T_{\text {sample }} \\
\text { in }{ }^{\circ} \mathrm{C}\end{array}$} \\
\hline & $\mathrm{Ga}$ & In & $\mathrm{N}$ & & & \\
\hline $\mathrm{T} 1$ & $\begin{array}{c}4.3 \\
(3.17)\end{array}$ & $\begin{array}{c}4.3 \\
(2.27)\end{array}$ & $\begin{array}{c}10 \\
(7.37)\end{array}$ & 0.74 & 0.72 & 750 \\
\hline $\mathrm{T} 2$ & $\begin{array}{c}4.3 \\
(3.17) \\
\end{array}$ & $\begin{array}{c}4.3 \\
(2.27) \\
\end{array}$ & $\begin{array}{c}10 \\
(7.37)\end{array}$ & 0.74 & 0.72 & 700 \\
\hline T3 & $\begin{array}{c}4.3 \\
(3.17)\end{array}$ & $\begin{array}{c}4.3 \\
(2.27)\end{array}$ & $\begin{array}{c}10 \\
(7.37)\end{array}$ & 0.74 & 0.72 & 650 \\
\hline T4 & $\begin{array}{c}4.3 \\
(3.17)\end{array}$ & $\begin{array}{c}4.3 \\
(2.27)\end{array}$ & $\begin{array}{c}14 \\
(10.3)\end{array}$ & 0.53 & 0.72 & 700 \\
\hline T5 & $\begin{array}{c}4.3 \\
(3.17) \\
\end{array}$ & $\begin{array}{c}6.3 \\
(3.33) \\
\end{array}$ & $\begin{array}{c}17 \\
(12.5)\end{array}$ & 0.52 & 1.05 & 700 \\
\hline T6 & $\begin{array}{r}2.3 \\
(1.7) \\
\end{array}$ & $\begin{array}{c}6.3 \\
(3.33) \\
\end{array}$ & $\begin{array}{c}14 \\
(10.3)\end{array}$ & 0.49 & 1.96 & 650 \\
\hline
\end{tabular}

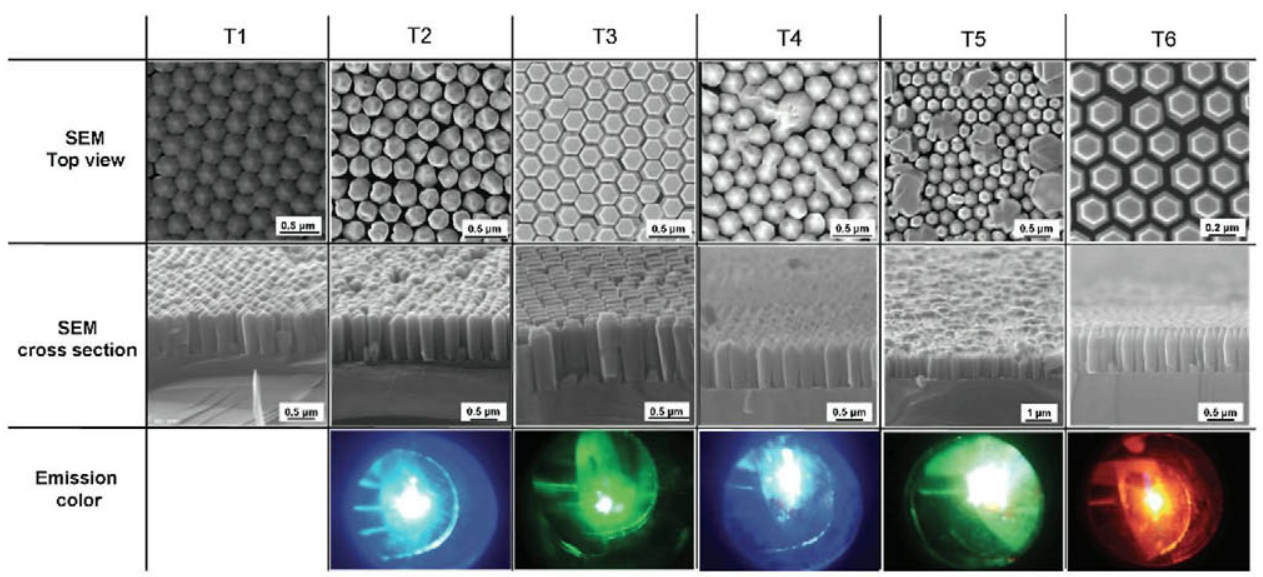

Fig. 5. SEM images (top view -upper row- and cross section -middle row-) and PL emission pictures (lower row) of samples of series A.

The SEM pictures in figure 5 shows the results from SAG of InGaN NCs in samples of series $\mathrm{A}$. The growth rates of the InGaN region, estimated from the cross-sectional SEM pictures, are summarized (together with the low temperature PL peak positions) in figure $6 a$. 
Similar to the GaN case discussed before, two main different top morphologies are found in figure 5, depending on the growth temperature and/or the III/V ratio. In the case of samples grown at the lower temperature (T3 and $\mathrm{T} 6$, at $650^{\circ} \mathrm{C}$ ), the top most facet is a flat c plane, while in the case of samples T1, T4 and T5, grown at $750{ }^{\circ} \mathrm{C}$ (T1) or $700^{\circ} \mathrm{C}$ (T4 and T5), pyramidal top facets are found. For sample T2, grown at the same temperature as T4 $\left(700^{\circ} \mathrm{C}\right)$, the top morphology looks like an irregular transition between pyramidal and flat, and can be understood because of its higher impinging III/V ratio compared to T4 (0.74 in $\mathrm{T} 2$ and 0.53 in T4).
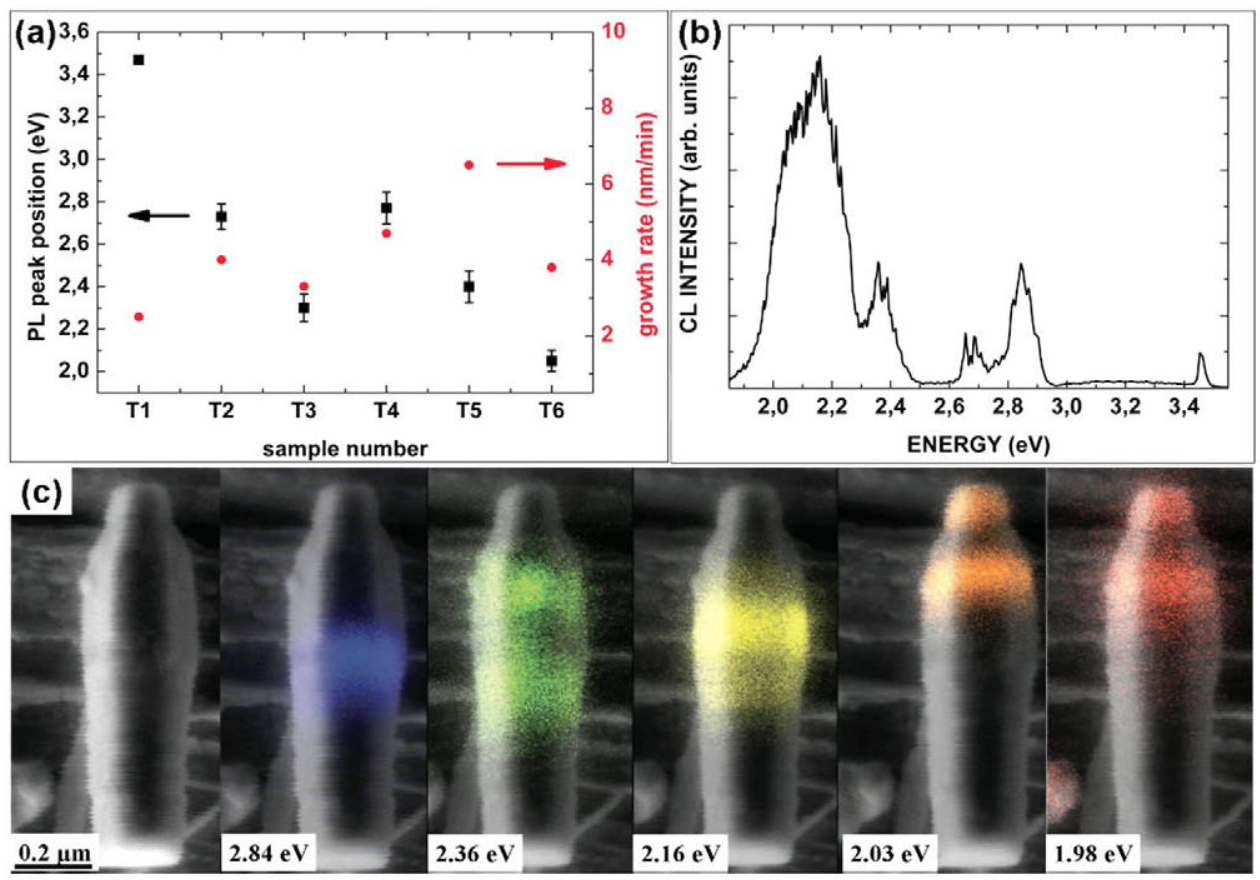

Fig. 6. a) Low temperature (12K) PL peaks and FWHM of InGaN/GaN NCs T1-T6 (black squares). Red circles represent the growth rate of the InGaN NCs regions; b) 10K CL spectra from a single InGaN/GaN NC of sample T6; c) Spatially resolved CL measurements at $10 \mathrm{~K}$ of a single InGaN/GaN NC from sample T6 (same single NC of spectra shown in figure 6b), adapted from J. Appl. Phys. 113, 114306 (2013).

Figure 6a shows the increase of growth rate (InGaN region) from sample T1 to T2 and a subsequent (slight) drop for sample T3, as growth temperature decreases from 750 to 700 and $650^{\circ} \mathrm{C}$ respectively (while keeping all other growth parameters constant). The total metal flux for the $\mathrm{InGaN}$ region, i.e. $\Phi_{\mathrm{Ga}}+\Phi_{\mathrm{In}}$, is assumed to be close to $\Phi_{\mathrm{Ga}}$ for sample $\mathrm{T} 1$ due to the very strong $\mathrm{InN}$ decomposition and In desorption rates at $750^{\circ} \mathrm{C}^{41}$. This is consistent with the presence of a $\mathrm{D}^{0} \mathrm{X}$ line at $3.472 \mathrm{eV}$ (FWHM of $6 \mathrm{meV}$ ) without traces of InGaN-related PL signal, indicating the growth of strain free GaN. In sample T2, grown at a lower temperature $\left(700^{\circ} \mathrm{C}\right)$, a reduced In desorption accounts for the observed increase of the growth rate. A further reduction of the growth temperature to $650^{\circ} \mathrm{C}$ yields an even lower In desorption but also a decrease of the Ga diffusivity that may account for the overall 
reduction of the InGaN growth rate. This interpretation is supported by the red shift of the PL peak position observed in samples T2 and T3 that indicates a higher In incorporation when decreasing the growth temperature. Similar results were previously reported in selfassembled InGaN/GaN NCs grown on $\mathrm{Si}(111)^{42}$. In addition it has to be noted that the same morphology transition as in case of GaN has been observed for InGaN (figure 5) i.e. transition from pyramid to flat top with decreasing temperature which corresponds to an increase of the local III/V ratio.

The PL peak trend observed in samples T4 to T6 reveal a continuous increase of In content, again coherent with either increasing the In/Ga ratio and decreasing the growth temperature. The InGaN growth rate in sample T5 further increases in respect to sample T4 because the increment of $\Phi_{\mathrm{N}}$, and finally decreases in sample T6 because of the low growth temperature (same argument as for sample T3).

It is worth noting that the PL peaks FWHM (indicated by vertical bars) shows no trend with In content, with these values being significantly smaller than those reported for self assembled InGaN/GaN NCs ${ }^{42}$, which suggests a much higher In\% uniformity on ordered NCs.

The LT-CL spectrum of a single NC from sample T6 is shown in figure $6 \mathrm{~b}$, where different peaks can be observed. Spatially resolved CL measurements performed in a single NC from sample T6 (figure 6c) reveal an emission red shift along the NCs growth direction concomitant with an In\% increase. Possible reasons for this are: i) $\operatorname{In}(\mathrm{Ga}) \mathrm{N}$ partial decomposition at $650^{\circ} \mathrm{C}$ with subsequent In segregation towards the growth front yielding a higher In\% towards the $\mathrm{NC}$ top ${ }^{41}$; ii) shadowing effect by NC diameter increase during the InGaN growth with a stronger reduction of the Ga supply by diffusion upwards along the NC sidewalls (higher effective In/Ga ratio) ${ }^{25}$; and iii) lower In incorporation at the beginning of the InGaN region to minimize strain effects due to mismatch, by which In atoms tend to segregate to more relaxed regions (lattice pulling) (self-regulated mechanism) ${ }^{43}$.

Once the emission wavelength was being controlled from red to blue, InGaN/GaN NCs emitting white light were addressed. A first approach was to use composition-graded InGaN regions by changing the growth temperature from $700^{\circ} \mathrm{C}$ to $650^{\circ} \mathrm{C}$ with a temperature gradient of $1.67^{\circ} \mathrm{C} / \mathrm{min}$. Sample $\mathrm{T} 7$ was grown with a continuously graded InGaN region, while $\mathrm{T} 8 \mathrm{had} \mathrm{GaN}$ insertions (barriers) at temperature points of $700^{\circ} \mathrm{C}$, $675^{\circ} \mathrm{C}$, and $650^{\circ} \mathrm{C}$ during the grading. In both cases, a broad LT-PL yields white light emission (figures $7 \mathrm{a}$ and $7 \mathrm{~b}$ ), though its intensity quenching at RT is higher and more pronounced at high energies in sample $\mathrm{T} 7$ (without GaN barriers). This quenching, also reported in self-assembled InGaN/GaN NCs ${ }^{42,44}$ is attributed to carrier diffusion to higher In\% regions (higher localization) upon thermal excitation. PL quenching in sample T8 is not that strong, also affecting the high energy spectral region, which is attributed to a partial blocking of carrier diffusion by the insertion of GaN barriers. This reduction of the intensity quenching leads to an increase of the internal quantum efficiency (LT versus RT PL intensity ratio) by a factor of 2 .

Spatially resolved CL measurements on a single NC from sample T7 (figure 7c) show a monotonic decrease of the emission energy from $2.98 \mathrm{eV}$ down to $2.12 \mathrm{eV}$ towards the 
NC top, as expected from a negative temperature gradient. The effects previously mentioned to explain emission broadening for single (nominal) composition InGaN regions, may also be present in this case, though the most effective one is the grading.
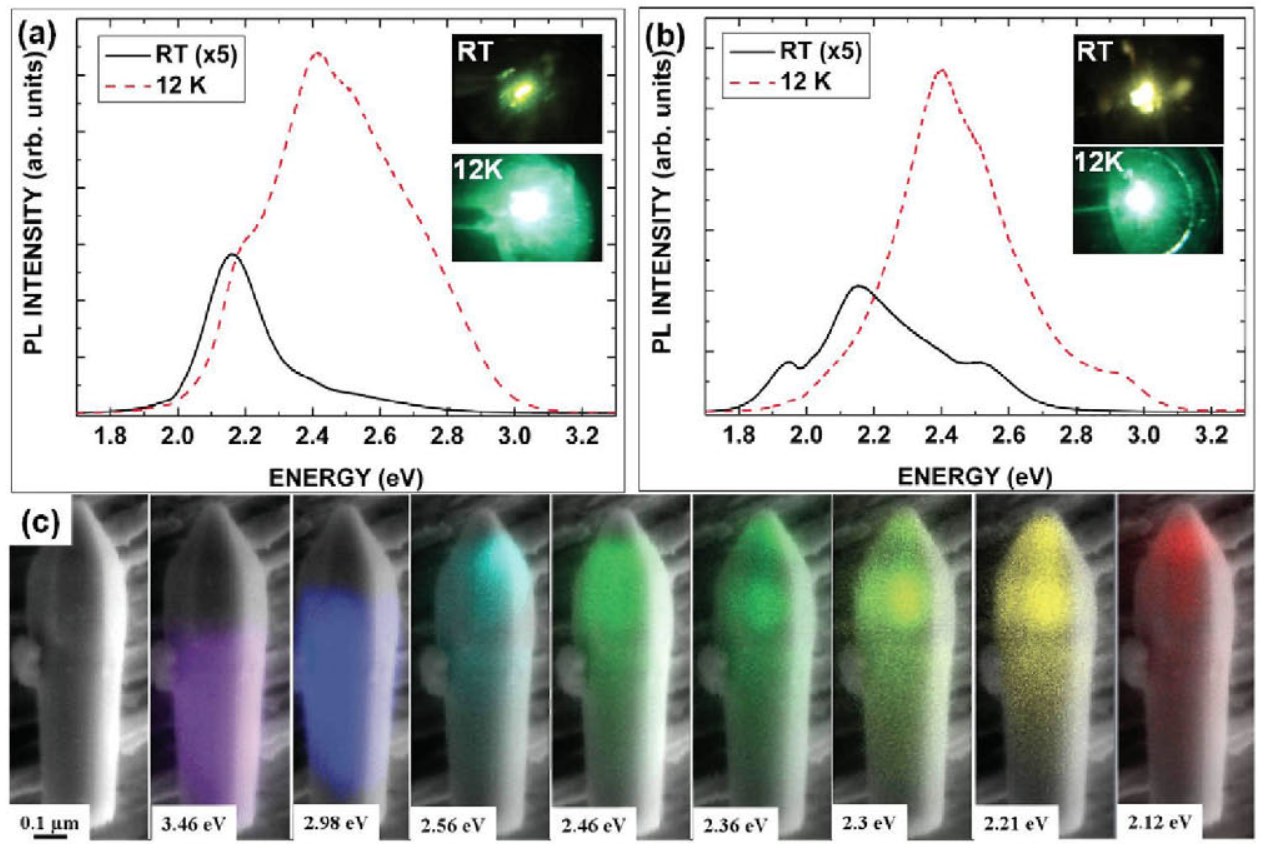

Fig. 7. PL spectra of InGaN/GaN NCs of samples T7 and T8 with graded In composition: a) without GaN barriers; b) with GaN barriers inserted at $700^{\circ} \mathrm{C}, 675^{\circ} \mathrm{C}$, and $650^{\circ} \mathrm{C}$ (insets show photographs of the emission color taken during PL experiments); c) $10 \mathrm{~K}$ spatially resolved CL emission from a single InGaN/GaN NC of sample T7, J. Appl. Phys. 113, 114306 (2013).

A drawback of the composition-graded approach is a rather low control of the emission spectrum which makes this approach unappealing for applications such as smart lighting.

Another approach that provides a higher control of the emission spectrum for white light emitters is to stack a sequence of InGaN regions with emission in the fundamental colors, red, green, and blue (RGB). Control of the "quality" of white (color temperature) can be achieved by tailoring the thickness of each InGaN region.

The RGB structure used stacked InGaN regions similar to those from samples T4 to T6 whose LT and RT PL spectra are shown in figure 8a. The In content estimated from the PL peak positions are $17 \%, 27 \%$ and $38 \%$ respectively. The PL spectra of sample T9 (an RGB full structure with 20 min growth for each InGaN region, figure 8 b) shows a blue-whitish emission at both RT and LT. However, sample T10 with InGaN sections of different thicknesses ( $8 \mathrm{~min}$ for the blue, $11 \mathrm{~min}$ for the green, and $41 \mathrm{~min}$ for the red) shows real white light primarily due to the enhancement of the red component (figure $8 \mathrm{c}$ ).

TEM measurements were performed to check the structure of T10 and determine the actual In\% distribution within different InGaN regions. 

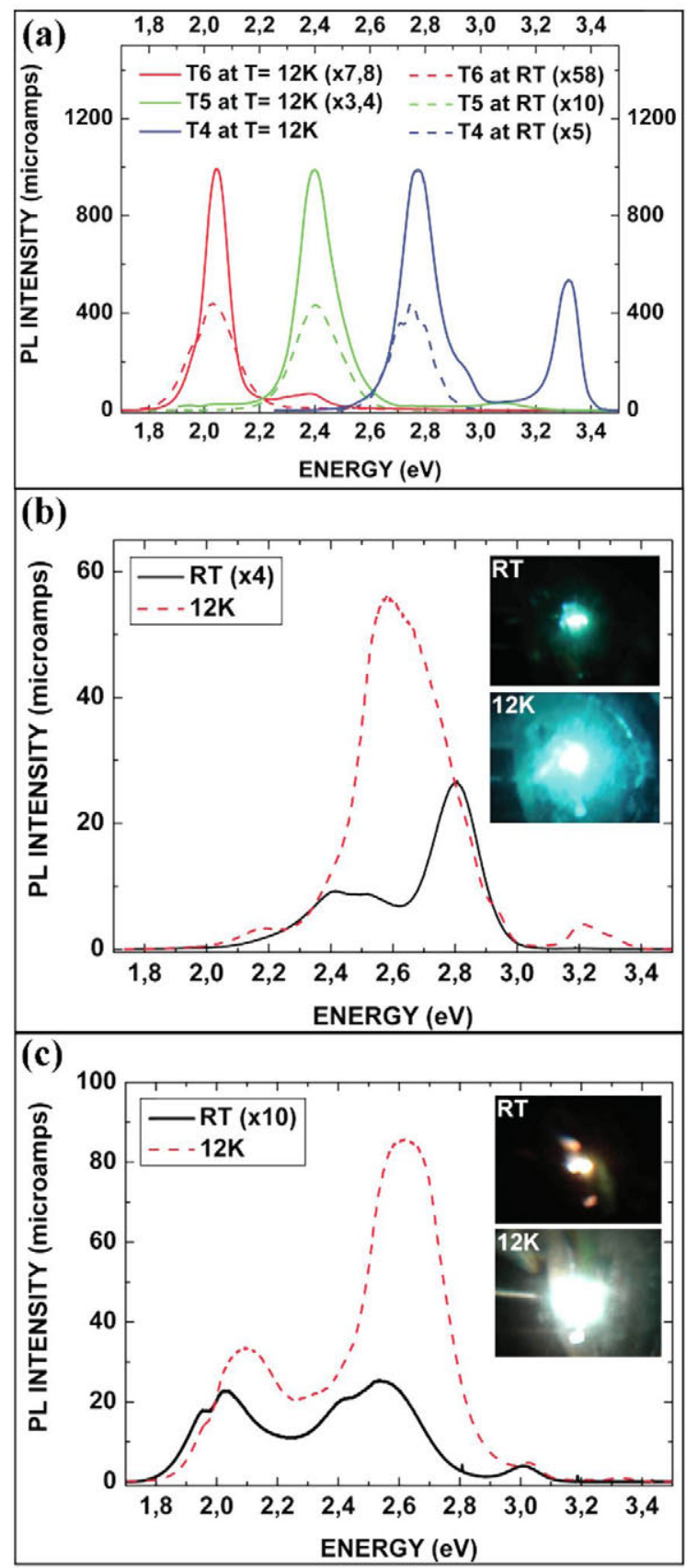

Fig. 8. RT and LT (12K) PL spectra of: a) samples T4, T5, T6; b) T9; and c) T10, from Appl. Phys. Lett. 102, 181103 (2013). 


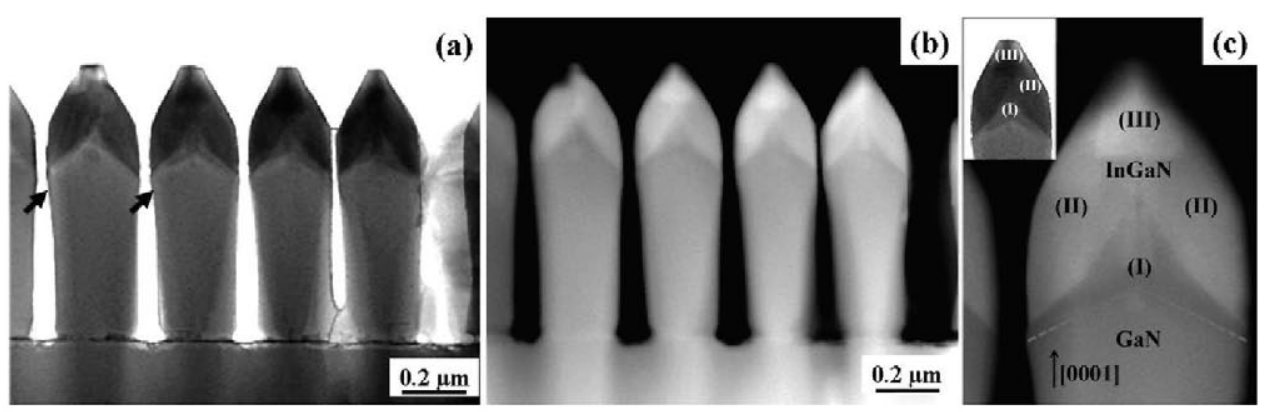

Fig. 9. (a) Bright-field STEM image of the NCs array of sample T10 in cross-section, (b) corresponding HAADF Z-contrast image identifying the InGaN regions on the upper part of the columns. (c) Magnified part of the top area with the different InGaN regions marked by I, II, and III (inset: appropriate bright-field STEM of the NC; dashed line corresponds to GaN/InGaN interface), Appl. Phys. Lett. 102, 181103 (2013).

Bright-field (S)TEM images in figure 9a demonstrate the high uniformity of the pencillike shaped NCs, as well as the absence of threading dislocations or other extended defects. A slight diameter increase can be observed at about the transition region between GaN and InGaN (indicated by the black arrows) which is identified by the dark strain contrast. The diameter increase is a clear indication for the elastic strain relaxation of the coherent InGaN. In order to resolve the different InGaN regions high-angle annular dark field (HAADF) STEM imaging (figure 9b and 9c) was used, where the contrast is directly correlated to the In content. As can be seen in figures $9 \mathrm{~b}$ and $9 \mathrm{c}$, three regions with distinct bright contrast are identified and are marked as (I), (II), and (III) in figure 9c. It has to be noted that well defined boundaries between the regions cannot be determined except for a semi-polar plane at the InGaN/GaN interface. The reason for these blurred transitions can be both a pulling effect that widens the interface region and the pencil-like morphology that results in an umbrella-shaped heterostructure which leads to overlapping between regions with different In content. Spatially-resolved low-loss EELS ${ }^{45}$ was carried out, along the axial and radial directions, on a single nanostructure from the same sample T10 to check the local In\% distribution.
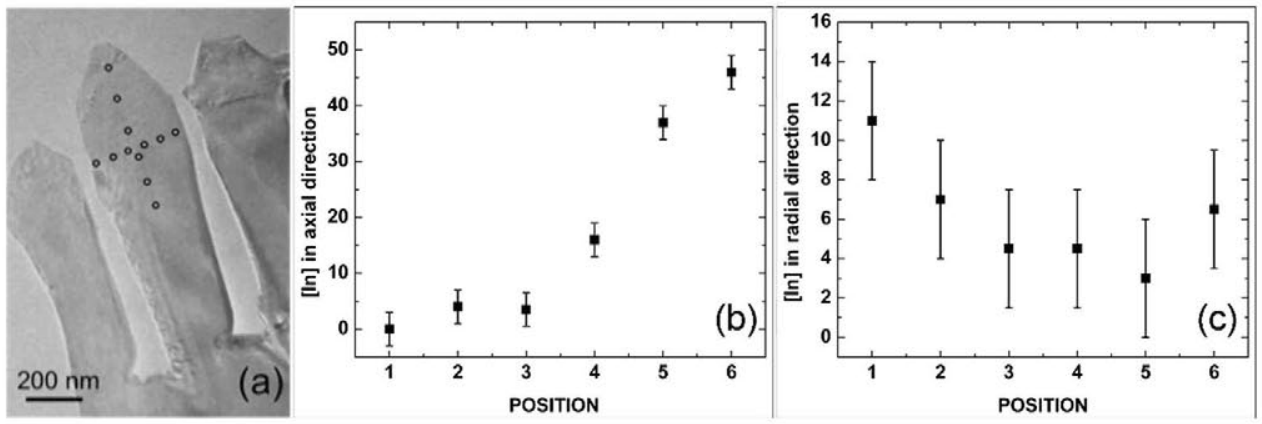

Fig. 10. Bright-field TEM image along [1-100] direction of a single InGaN/GaN nanorod (a), and axial (b) and radial (c) low-loss EELS scans as indicated by dots in the bright-field image, Appl. Phys. Lett. 102, 181103 (2013). 